\title{
A Novel Approach in Sample Preparation of Li Content Materials for TEM Research
}

\author{
Sz-Chian Liou ${ }^{1}$, Chuan-Fu Lin ${ }^{2}$, Wen-An Chiou ${ }^{1}$ and Gary Rubloff ${ }^{2}$ \\ 1. AIM Lab, NanoCenter, University of Maryland, College Park, MD, U.S.A \\ 2. Dept. of Materials Science and Eng., University of Maryland, College Park, MD, U.S.A
}

Lithium ( $\mathrm{Li}$ )-ion rechargeable batteries have attracted world-wide interest due to their high energy density, tiny memory effect, and low maintenance for powering portable electronics, hybrid electric vehicles, and aerospace applications. To improve battery efficiency and life time, understanding the microstructure evolution at electrode/electrolyte interface during charge/discharge (conversion reaction) processes is critical [1]. However, due to high reactivity of Li to moisture, TEM specimen preparation for Li content materials has been challenging. Focused ion beam (FIB) technology has thus become the most popular method to prepare TEM specimen for Li-ion materials. Furthermore, porous solid-state electrolyte, e.g. lithium phosphorous oxynitride (LiPON), is susceptible to damage by ion beam bombardment during milling [2]. To fully characterize the moisture and ion beam sensitive interface (LiPON/FeOF), a unique approach by combing FIB technique and ultra-microtome was performed and evaluated.

An electrode protective layer (LiPON) was deposited on the FeOF/CB (carbon black) composites and polyvinylidene difluoride (PVDF) binder by ALD [3]. Samples were kept in vacuum until TEM specimen preparation. Thin TEM specimens were prepared by a FIB/SEM (Tescan GAIA), and were immediately transferred into the TEM column ( $<5$ mins.) with special care to minimize possible $\mathrm{Li} / \mathrm{H}_{2} \mathrm{O}$ reaction in air. To investigate the sample in $3 \mathrm{D}$, samples before discharge process were embedded in Spurr low viscosity resin and cured at $70{ }^{\circ} \mathrm{C}$ for overnight. TEM thin specimens $(\sim 80 \mathrm{~nm}$ in thickness $)$ were cut with an ultramicrotome (Reichert Ultracut E) using a DiATOME diamond knife. The distribution and concentration of constitute elements were analyzed using ToF-SIMS attached on the $\mathrm{Ga}^{+}$FIB/SEM. Microstructure and elemental composition were examined using JEOL 2100F TEM/STEM equipped with Gatan Tridiem 863 EELS/GIF and Oxford EDS systems. Electron tomography was performed using JEOL $2100 \mathrm{LaB}_{6} \mathrm{TEM}$ Images (134) for 3D reconstruction were collected between $\pm 67^{\circ}$ with one image per degree. Tomographic reconstruction and visualization were carried out using TEMography software (System in Frontier, Inc.).

Cross-sectional TEM images and SAD patterns of LiPON coated FeOF/CB composites (before discharge) prepared by ultra-microtome clearly showed well preserved porous layer ( $\sim \mu \mathrm{m}$ in thickness) that consisted of dispersed FeOF nano-tetrahedron and nano-rods of different lengths/sizes (open arrows) mixed with very small round and/or spherical CB particles (arrows) without pure metallic Fe (Fig. 1). EELS mapping depicted well-coated LiPON on FeOF/CB composites. Although the coated electrode layer detached from the Al substrate due to differential stress while microtoming, the ultra-microtomed specimens provided a large overall view of the sample (>500 $\mu \mathrm{m}$ in length) and allowed for 3D reconstruction which illustrated a homogeneous distribution of FeOF and CB particles in the porous electrode (Fig. 2). Results of FIB/SEM observation (top- and cross-sectional-view) of the same sample were consistent with TEM study. ToF-SIMS also revealed that the majority of Li localized in the top layer and diffused into FeOF/CB composites for $1 \sim 2 \mu \mathrm{m}$ in depth (Fig. 3). TEM images of LiPON protected FeOF/CB electrode after discharge obtained from FIB prepared specimen showed unaltered tetrahedral/rod-like morphology of FeOF. In contrast, drastic morphology change and pure Fe phase were found in the unprotected electrode after discharge (Fig. 4). This study introduced a unique approach in preparing porous Li content materials by combining ultra-microtome and FIB, and documented the effectiveness of LiPON in protecting FeOF/CB from conversion reaction [4]. References:

[1] M. Saiful Islam and C. A. J. Fisher, Chem. Soc. Rev. 43 (2014), p. 185.

[2] Z. Wang and Y. S. Meng in Handbook of Solid State Batteries, N. J. Dudney et al, ed., (2015), p. 109.

[3] X. Fan et al, Nano Letters 15(11) (2015), p.7650; and A. C. Kozen et al, Chem. Mater. 27, (2015) p.5324. 
[4] Research was supported by Nanostructures for Electrical Energy, DOE (DESC0001160) and UMD.

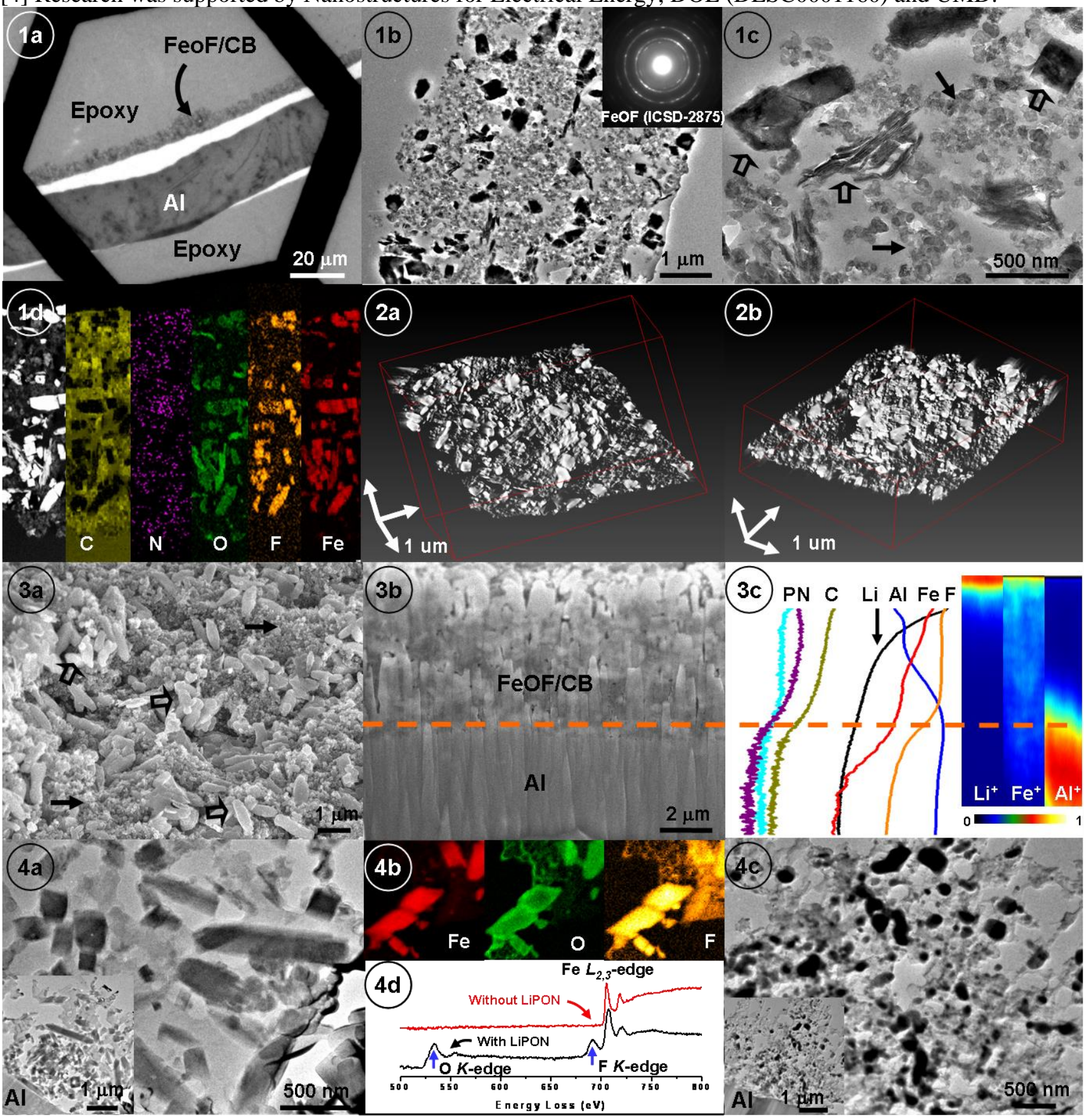

Figure 1. TEM images and SAD pattern of LiPON coated FeOF/CB composites (before discharge) prepared by ultra-microtome showed tetrahedron- and rod-like FeOF (open arrows) and round/spherical CB particles (arrows) (a to c). Nitrogen in EELS mapping depicted LiPON coated on FeOF/CB (d).

Figure 2. Reconstructed 3D TEM images illustrated porous FeOF/CB electrode coated with LiPON.

Figure 3. Top-view (a) and FIB cross-sectional (b) SEM images of LiPON coated FeOF/CB composites showed FeOF (elongated rod-like, open arrows) and CB (round/spherical, arrows) particles. ToF-SIMS analysis depicted elemental mapping of $\mathrm{Li}, \mathrm{Fe}$ and $\mathrm{Al}$ ions and depth profiles of various ions in the sample, which showed that LiPON was well-deposited/diffused into FeOF/CB electrode (c).

Figure 4. TEM images and EELS results showed morphological and chemical change of FeOF/CB electrode with and without LiPON layer after discharge prepared by FIB (a/b and c/d, respectively). 